\title{
SARCOMA PLEOMÓRFICO GIGANTE DE LA MAMA*
}

\author{
Drs. Francisco Herrera S. ${ }^{1,2,3}$, Katherine Redondo D. ${ }^{3,4,5}$, \\ Eduardo Vega E. ${ }^{6}$, Al. Carlos Osorio C. ${ }^{3,7}$, Alberto Fernández D. ${ }^{3,7}$, \\ Pedro Caraballo G. ${ }^{3,7}$, Ruben Agresott M.,
}

1 Sección de Cirugía General. Facultad de Medicina. Universidad de Cartagena.

2 Servicio de Cirugía de Cuello y Mama. E.S.E. Hospital Universitario del Caribe.

3 Grupo de Investigación Scalpellum.

4 Sección de Patología. Facultad de Medicina. Universidad de Cartagena.

5 Servicio de Citopatología. E.S.E. Hospital Universitario del Caribe.

6 Servicio de Cirugía Plástica. E.S.E. Hospital Universitario del Caribe.

7 Estudiante de pregrado en Medicina. Facultad de Medicina. Universidad de Cartagena.

8 Estudiante de posgrado en Cirugía General. Facultad de Medicina. Universidad de Cartagena. Cartagena, Colombia.

Abstract

\section{Giant pleomorphic breast sarcoma}

It is reported the case of a female patient 50 years old who, after failed attempts at diagnosis, arrives at breast surgery service of the E.S.E. Hospital Universitario del Caribe with history of five months consistent in the excessive growth of a mass in right breast; patient received radical mastectomy plus reconstruction with latissimus dorsi muscle flap. Following excision of tumor mass of $6,500 \mathrm{~g}$ is performed immunohistochemical diagnosis of a giant pleomorphic sarcoma.

Key words: Pleomorphic sarcoma, breast.

\section{Resumen}

Introducción: Los tumores derivados del tejido conectivo interlobulillar de la mama son en mayor proporción malignos y, en virtud a su ubicación, en la actualidad reciben el nombre de "sarcomas" de la mama. Caso clínico: Se reporta el caso de una paciente femenina de 50 años de edad quien, posterior a fallidos intentos de diagnóstico, acude al servicio de cirugía de mama de la E.S.E. Hospital Universitario del Caribe con un cuadro de cinco meses de evolución consistente en el crecimiento desmedido de una masa en mama derecha; la paciente recibió mastectomía radical más reconstrucción con colgajo de músculo dorsal ancho. Posterior a exéresis de masa tumoral de $6.500 \mathrm{~g}$ de peso se lleva a cabo diagnóstico inmunohistoquímico de un sarcoma pleomórfico gigante de la mama.

Palabras clave: Sarcoma pleomórfico, tumor del estroma mamario.

*Recibido el 17 de mayo de 2015 y aceptado para publicación el 11 de agosto de 2015.

Los autores no refieren conflictos de interés.

Correspondencia: Francisco Herrera S.

fherreras@gruposcalpellum.com 


\section{Introducción}

Dependiendo del tipo de tejido conectivo del cual se originan, los tumores del estroma mamario se dividen en tumores de origen intralobulillar o interlobulillar $^{1}$. Los primeros reciben el nombre de tumores bifásicos ya que secretan factores de crecimiento que estimulan la proliferación de un componente epitelial no neoplásico, siendo integrantes exclusivos de este grupo el fibroadenoma y el tumor phyllodes de la mama ${ }^{2,3}$. Por otro lado, los tumores derivados del tejido conectivo interlobulillar son similares a los encontrados en el tejido conectivo de otras regiones corporales, siendo en mayor proporción malignos, en virtud a su ubicación en la actualidad reciben el nombre de sarcomas de la mama ${ }^{4}$.

Estos tumores pueden ser primarios o secundarios. Los primarios, también llamados sarcomas de novo, son aquellos que no poseen un factor causal claro, mientras que los secundarios son aquellos en los que se demuestra el antecedente de exposición a radiación como tratamiento de una neoplasia maligna ${ }^{5}$. Entre el grupo de sarcomas mamarios, el sarcoma pleomórfico, antiguamente denominado fibrohistiocitoma maligno, es el segundo más frecuente, con una frecuencia diagnóstica que oscila entre un 10 y un $25 \%{ }^{6}$.

Presentamos el caso de una paciente con el diagnóstico de un sarcoma pleomórfico gigante primario, la cual fue sometida a mastectomía radical modificada más reconstrucción con colgajo de músculo dorsal ancho.

\section{Presentación del caso}

Paciente de 50 años de edad, residente y procedente de la ciudad de Cartagena-Colombia, quien consulta al servicio de cirugía de mama de la E.S.E. Hospital Universitario del Caribe por un cuadro clínico de cinco meses de evolución, caracterizado por el crecimiento desmedido de una lesión tumoral en mama derecha. Paciente refiere manejo extrainstitucional, siendo sometida a numerosas biopsias por aspiración con aguja fina, las cuales fueron reiterativamente insuficientes para diagnóstico.

$\mathrm{Al}$ ingreso se realiza examen físico en el que se evidencia mama derecha con aumento considerable de su tamaño, reemplazada por una lesión tumoral ubicada en cuadrantes superior e inferior derecho con francas zonas de induración, además se observa solución de continuidad de $11 \mathrm{~cm}$ de diámetro ubicada entre las 7 y las $10 \mathrm{~h}$ de reloj, asociada a una amplia zona de necrosis; no se evidencia secreción activa o pasiva por pezón y no se palpan adenopatías axilares ni supraclaviculares (Figura 1).
Debido al rápido crecimiento de la lesión y a la ausencia de adenopatías palpables, se hace la impresión clínica de un tumor de origen mesenquimal y se procede a la realización de una biopsia incisional para llevar a cabo un diagnóstico preoperatorio. Esta reporta hallazgos histoarquitecturales correspondientes a un sarcoma del estroma mamario. Basado en lo anterior se decide llevar a la paciente a mastectomía radical modificada sumada a reconstrucción con colgajo dorsal.

Se lleva a cabo procedimiento quirúrgico con exéresis de la masa tumoral, y desinserción de músculos pectoral mayor y pectoral menor (Figuras 2 y 3). En el mismo acto quirúrgico se realiza reconstrucción mediante translocación de colgajo de músculo dorsal ancho e injerto de piel (Figura 4). Finalizado el procedimiento, la paciente es hospitalizada para manejo antibiótico $\mathrm{y}$, posterior a una evolución satisfactoria del colgajo, es dada de alta.

La paciente es valorada en el servicio de consulta externa, al cual acude con el resultado de la evaluación anatomopatológica del espécimen quirúrgico, en donde se evidenció una mama totalmente reemplazada por una lesión tumoral de $6,5 \mathrm{~kg}$ de peso, con dimensiones de $31 \times 26$ × $18 \mathrm{~cm}$ (Figura 5). Los cortes histológicos muestran una lesión mesenquimal constituida por la proliferación de células grandes, de núcleos hipercromáticos, marcado pleomorfismo y citoplasmas intensamente eosinófilos, que se disponen formando un patrón arremolinado, acompañado de abundantes figuras de mitosis atípicas y extensas áreas de necrosis; por todo lo anterior se realiza el diagnóstico de un sarcoma del estroma mamario de alto grado y se solicitan estudios de inmunohistoquímica para determinar la histogénesis de la lesión.

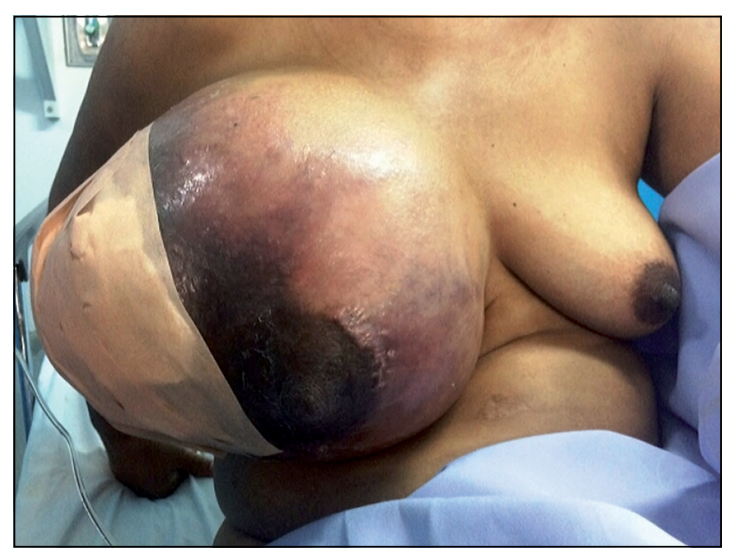

Figura 1. Comparación de las características evidenciadas al examen físico del seno comprometido por la lesión tumoral en relación al seno contralateral sano. 


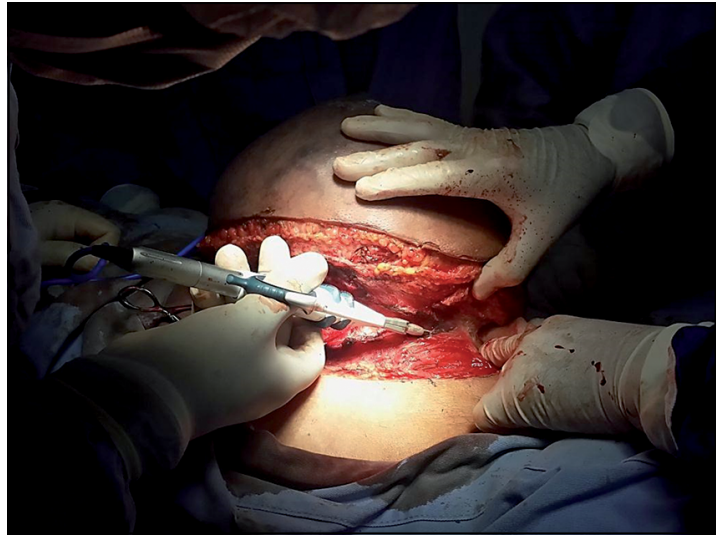

Figura 2. Evolución intraoperatoria de la paciente. Exéresis de lesión tumoral en seno derecho.

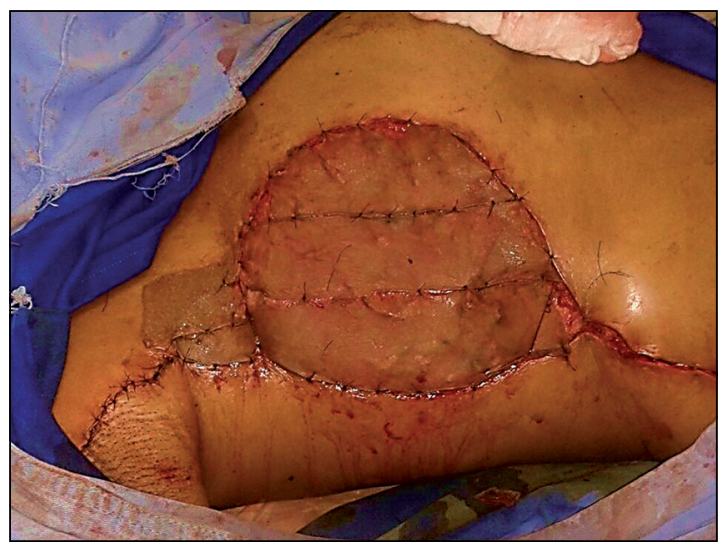

Figura 4. Evolución intraoperatoria de la paciente. Inserción de colgajo correspondiente a músculo dorsal ancho e injertos de piel.

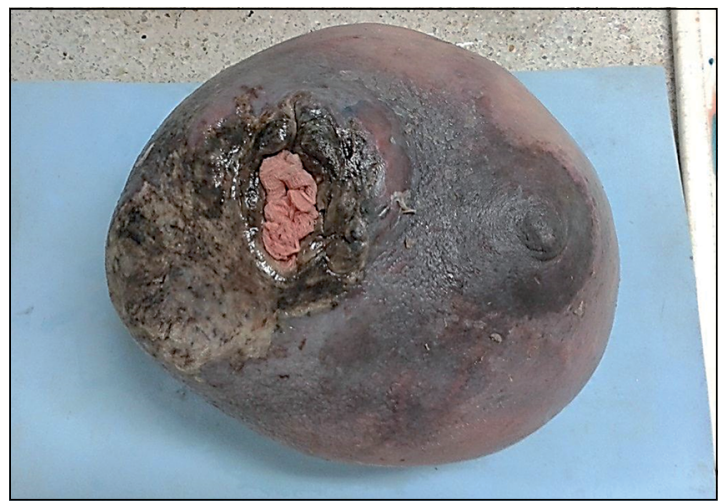

Figura 5. Pieza quirúrgica correspondiente a mastectomía derecha. Peso de masa tumoral: $6.500 \mathrm{~g}$; dimensiones de masa tumoral: 310 × $260 \times 180 \mathrm{~mm}$.

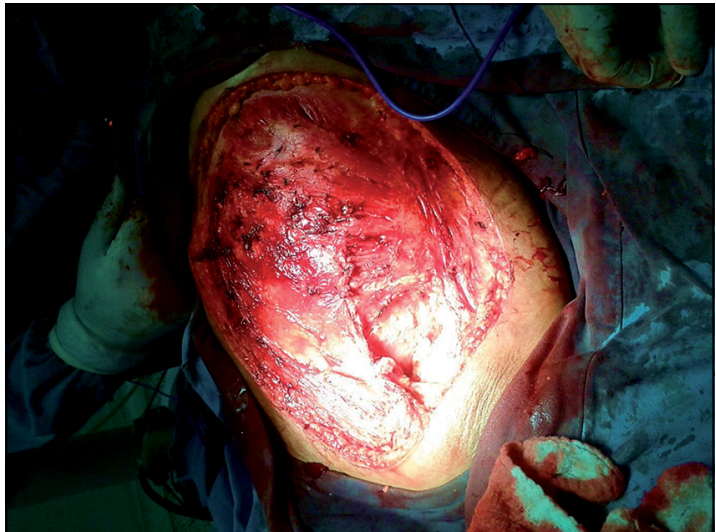

Figura 3. Evolución intraoperatoria de la paciente. Sitio operatorio posterior a la desinserción de los músculos pectorales y al vaciamiento ganglionar.

Un mes después, la paciente acude con resultado del estudio inmunohistoquímico, en el cual se evidencia una lesión tumoral maligna mal diferenciada en la que no se reconocen elementos epiteliales, cuyas células tumorales muestran reactividad para vimentina y un índice de proliferación celular medido con ki-67 del 73\%, no hay reactividad para antígeno epitelial de membrana (EMA), actina de músculo liso, CD34, CD117 ni S100, por lo cual se torna imposible determinar claramente la histogénesis del tumor, llegando al diagnóstico definitivo de un sarcoma pleomórfico de alto grado. Se confirma la evolución satisfactoria del colgajo (Figura 6) y la paciente es remitida al servicio de oncología clínica de la institución para inicio del manejo médico.

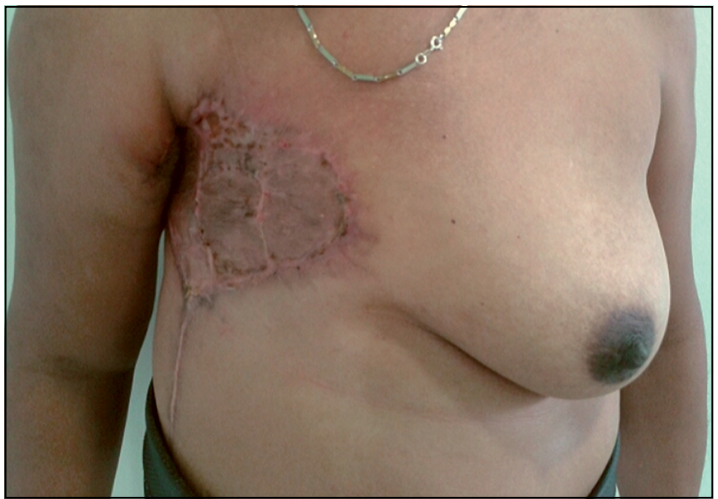

Figura 6. Evolución post quirúrgica de la paciente. Se evidencia colgajo de músculo dorsal ancho en excelentes condiciones. 
Tabla 1. Características clínicas del total de casos con diagnóstico inmunohistoquímico de sarcoma pleomórfico de la mama

\begin{tabular}{|c|c|c|c|c|c|c|c|c|}
\hline Año & Autor & Edad & Evolución & Tamaño & Ubicación & Procedimiento & Ganglios & Metástasis \\
\hline 2004 & Jeong et $\mathrm{al}^{9}$ & 48 & 9 meses & $13 \mathrm{~cm}$ & Mama izquierda & $\begin{array}{l}\text { Mastectomía } \\
\text { radical }\end{array}$ & Reactivos & Pulmonar \\
\hline 2005 & Yao et $\mathrm{al}^{10}$ & 46 & 3 meses & $06 \mathrm{~cm}$ & Mama derecha & $\begin{array}{l}\text { Mastectomía } \\
\text { radical }\end{array}$ & Negativos & - \\
\hline 2007 & Kilima et $\mathrm{al}^{11}$ & 56 & $1 \mathrm{mes}$ & $05 \mathrm{~cm}$ & Mama derecha & Cuadrantectomía & Negativos & - \\
\hline 2010 & Choudhury et al ${ }^{12}$ & 32 & 8 meses & $09 \mathrm{~cm}$ & Mama derecha & $\begin{array}{l}\text { Mastectomía } \\
\text { radical }\end{array}$ & Negativos & - \\
\hline 2010 & De Mello et $\mathrm{al}^{13}$ & 42 & 7 meses & $03 \mathrm{~cm}$ & Mama izquierda & $\begin{array}{l}\text { Mastectomía } \\
\text { radical }\end{array}$ & - & - \\
\hline 2012 & Miliaras et al $^{14}$ & 73 & 2 meses & $02 \mathrm{~cm}$ & Mama derecha & Cuadrantectomía & Negativos & - \\
\hline 2013 & Chakrabarti el al ${ }^{15}$ & 60 & - & $06 \mathrm{~cm}$ & Mama izquierda & $\begin{array}{l}\text { Mastectomía } \\
\text { radical }\end{array}$ & - & Cerebral \\
\hline 2013 & Liu et $\mathrm{al}^{16}$ & 48 & 2 meses & $07 \mathrm{~cm}$ & Mama derecha & $\begin{array}{l}\text { Mastectomía } \\
\text { radical }\end{array}$ & - & Pulmonar \\
\hline 2013 & Tashjian et al ${ }^{17}$ & 72 & - & $03 \mathrm{~cm}$ & Mama izquierda & $\begin{array}{l}\text { Mastectomía } \\
\text { radical }\end{array}$ & - & Pulmonar \\
\hline 2013 & Balbi et al ${ }^{18}$ & 50 & $1 \mathrm{mes}$ & $19 \mathrm{~cm}$ & Mama derecha & $\begin{array}{l}\text { Mastectomía } \\
\text { radical }\end{array}$ & - & - \\
\hline 2014 & Thomas et al ${ }^{19}$ & 45 & 5 meses & $12 \mathrm{~cm}$ & Mama derecha & $\begin{array}{l}\text { Mastectomía } \\
\text { radical }\end{array}$ & Negativos & - \\
\hline 2015 & Presente reporte & 50 & 5 meses & $31 \mathrm{~cm}$ & Mama derecha & $\begin{array}{l}\text { Mastectomía } \\
\text { radical }\end{array}$ & Negativos & - \\
\hline
\end{tabular}

\section{Discusión}

Los esbozos del actualmente denominado sarcoma pleomórfico fueron descritos por primera vez en 1961 por Kauffman \& Stout, quienes le dieron su antigua denominación de fibrohistiocitoma maligno, esto en virtud a que las células tumorales poseían la apariencia de histiocitos con un patrón de crecimiento arremolinado ${ }^{7}$. En 2002, la Organización Mundial de la Salud eliminó el término fibrohistiocitoma maligno como una categoría de diagnóstico, dando la denominación de sarcoma pleomórfico al conjunto de lesiones de origen mesenquimal que experimentan una evolución progresiva a la desdiferenciación ${ }^{8}$.

$\mathrm{Al}$ analizar los casos reportados en la literatura (Tabla 1), los cuales poseen diagnóstico inmunohistoquímico confirmatorio ${ }^{9-19}$, los sarcomas pleomórficos de la mama se presentan clínicamente como una masa unilateral de consistencia firme y márgenes bien definidos a la palpación; se distinguen clínicamente de los carcinomas mamarios por su rápido patrón de crecimiento, y porque que a pesar de su gran tamaño, no existe relación entre este y el compromiso de ganglios, lo cual es fundamental al momento de establecer un diagnóstico diferencial ${ }^{20}$.

Es muy común que los sarcomas de la mama, en el contexto de un desmedido tamaño tumoral, se asocien a soluciones de continuidad que generan úlceras tendientes a la sobreinfección, como es el caso de nuestra paciente. Por el contrario, los carcinomas de la mama, independientemente de su tamaño, son capaces de invadir localmente la piel, siendo muy común el compromiso del complejo areola pezón, dicho comportamiento es infrecuente en los sarcomas de la mama ${ }^{21}$. Por tal razón, pese a que la biopsia por aspiración con aguja fina es considerada la herramienta de elección al momento de llevar a cabo un diagnóstico preoperatorio, son las características clínicas quienes deben hacer sospechar el diagnóstico.

El manejo quirúrgico de estas lesiones es actualmente discutido, pero sin lugar a duda el procedimiento más recomendado en la actualidad es la mastectomía radical $^{22}$. El objetivo principal de este abordaje consiste en realizar exéresis total de la masa tumoral, puesto que las recidivas locales son 
el principal temor durante el seguimiento y representan el factor que más afecta la sobrevida de los pacientes $^{23}$. Por último, en virtud a la casi nula tasa de metástasis linfáticas, el vaciamiento axilar no está indicado en el tratamiento de este tipo de tumores ${ }^{24}$.

La respuesta de los sarcomas de la mama al uso de quimioterapia es poco favorable ${ }^{25}$, razón por la cual, actualmente se considera la radioterapia como el tratamiento de elección, pues autores han demostrado que está relacionado con una mayor sobrevida y un período libre de enfermedad más prolongado ${ }^{20}$.

\section{Referencias}

1. Berg JW, Hutter RV. Breast cancer. Cancer 1995;75(1 Suppl):257-69.

2. Dupon WD, Page DL, Parl FF, Vnencak CL, Plummer WD, Rados MS, et al. Long term risk of breast cancer in women with fibroadenoma. N Engl J Med. 1994:331:10-5.

3. Telli ML, Horst KC, Guardino AE, Dirbas FM, Carlson RW. Phyllodes tumors of the breast: natural history, diagnosis and treatment. J Natl Compr Canc Netw. 2007;5:324-30.

4. Schickman R, Leibman A, Handa P, Kornmehl A, Abadi M. Mesenchymal breast lesions. Clinical Radiology 2015;70:567-75.

5. Sheth GR, Cranmer LD, Smith BD, Grasso-LeBeau L, Lang JE. Radiation-induced sarcoma of the breast: a systematic review. The Oncologist 2012;17:405-18.

6. Adem C, Reynolds C, Ingle JN, Nascimento AG. Primary breast sarcoma: clinicopathologic series from the Mayo Clinic and review of the literature. Br J Cancer 2004;91:237-41.

7. Kauffman SL, Stout AP. Histiocytic tumors (fibrous xanthoma and histiocytoma) in children. Cancer 1961;14:469-82.

8. Fletcher CD, Unni KK, Mertens F (Eds): World Health Organization Classification of Tumors. Pathology and Genetics of Tumors of Soft Tissue and Bone. IARC Press: Lyon 2002.

9. Oh SJ, Kim KM, Hong TH, Park WC, Kim JS, Jung SS. Giant Cell Malignant Fibrous Histiocytoma of the Breast: a case report. J Korean Med Sci. 2004;19:47780.

10. Yao MS, Chan WP, Chen CY, Chu JS, Hsieh MC. Malignant fibrous histiocytoma of the breast: a case report. Journal of Clinical Imaging 2005;29:134-7.

11. Kijima Y, Umekita Y, Yoshinaka H, Taguchi S, Owaki T, Funasako Y, et al. Stromal Sarcoma with Features of Giant Cell Malignant Fibrous Histiocytoma. Breast Cancer 2007; 14:239-44.

12. Choudhury M, Nangia A, Singh SK, Pujani M, Thomas S. Cytohistomorphologic Features of Malignant Fibrous Histiocytoma of the Breast: a case report. Acta Cytol.
2010;54(5 Suppl):985-8.

13. De Mello R, Figueiredo P, Marques M, Sousa G, Carvalho $\mathrm{T}$, Gervasio $\mathrm{H}$. Concurrent breast stroma sarcoma and breast carcinoma: a case report. Journal of Medical Case Reports 2010. Online reference: http://www.jmedicalcasereports.com/content/4/1/414

14. Miliaras D, Konstantinides E. Malignant Fibrous Histiocytoma of the Breast: a case report. Case Reports in Pathology 2012;1:1-3.

15. Chakrabarti I, Ghosh N, Giri A. Cytologic diagnosis of undifferentiated high grade pleomorphic sarcoma of breast presenting with brain metastasis. Journal of neurosciences in rural practice 2013;4:188-90.

16. Caigang L, Zuowei Z, Qingfu Z, Yunfei W, Feng J. Primary malignant fibrous histiocytoma of the breast: report of one case. Oncotarget and therapy 2013;6:3159.

17. Tashjian R, Gilani SM, Falk J, Kelly MJ, Ockner D, Danforth R. Primary inflammatory malignant fibrous histiocytoma of the breast: a case report of an unusual variant and review of the literature. Pathology research and practice.2013;209:534-9.

18. Balbi G, Di Martino L, Pitruzzella G, Pitruzella D, Grauso F, Napolitano A, et al. Undifferentiated Pleomorphic sarcoma with osteoclast-like giant cells of the female breast. World journal ol surgical oncology 2013;11:21-7.

19. Suhani, Thomas S, Ali S, Choudhary M, Deswal V, Aggarwal L. Denovo malignant fibrous histiocytoma of breast: report of a case and review of literature. Breast Diseases 2014;34:135-8.

20. Gutman H, Pollock RE, Ross MI, Benjamin RS, Johnston DA, Janjan NA, et al. Sarcoma of the breast: implications for extent of therapy. The MD Anderson experience. Surgery 1994;116:505-9.

21. Pollard SG, Marks PV, Temple LN, Thompson HH. Breast sarcoma. A clinicopathologic review of 25 cases. Cancer 1990; 66:941-5.

22. Shabahang M, Franceschi D, Sundaram M, Castillo MH, Moffat FL, Frank DS, et al. Surgical management of primary breast sarcoma. The American Surgeon. 2002;68:673-7.

23. Zelek L, Llombart-Cussac A, Terrier P, Pivot X, Guinebretiere J, Le Pechoux C, et al. Prognostic factors in primary breast sarcomas: a series of patients with long-term follow-up. Journal of Clinical Oncology 2003;21:2583-8.

24. Gullett NP, Delman K, Folpe AL, Johnstone PA. National surgical patterns of care: regional lymphadenectomy of breast sarcomas. American journal of clinical oncology 2007;30:461-5.

25. Bousquet G, Confavreux C, Magné N, de Lara CT, Poortmans P, Senkus E, et al. Outcome and prognostic factors in breast sarcoma: a multicenter study from the rare cancer network. Radiotherapy and Oncology 2007;85:355-61. 